\title{
Familial Hypocalciuric Hypercalcaemia (FHH): A Case Report
}

\author{
Tivya Kulasegaran ${ }^{1}$, Pranav Kumar ${ }^{2}$ \\ ${ }^{1}$ Medical Registrar, Prince Charles Hospital, Queensland Health, Australia \\ ${ }^{2}$ FRACP, Respiratory Physician, Mackay Base Hospital, Queensland Health, Australia
}

*Corresponding Author:

Dr. Pranav Kumar

Mackay Base Hospital

475 Bridge Road

Mackay, Queensland Australia 4740

\section{Introduction}

Familial hypocalciuric hypercalcaemia $(\mathrm{FHH})$ is a rare genetic autosomal dominant disorder, with 3 variants described. An inactivating mutation in the calcium sensor receptor (CASR) gene causes the subtype 1, which represents $65 \%$ of the cases. Inactivation of Ca-sensing receptors (CaSR) can also lead to hypercalcemia associated with increased parathyroid hormone (PTH) secretion. ${ }^{[1]}$

It is characterised by causes mild asymptomatic hypercalcemia ${ }^{[2]}$ and hypocalciuria with normal or elevated PTH. FHH is generally asymptomatic and treatment is not needed. Differential diagnosis with primary hyperparathyroidism (PHPT) is crucial and based on calcium-creatinine clearance ratio (CCCR), which, when under 0.02 points to the diagnosis of FHH. ${ }^{[3]}$ Genetic test is necessary for confirmation. ${ }^{[4]}$

\section{Case Presentation}

The proband (Fig. 1), a 60 year old female, was initially admitted to hospital with poor mobility and poorly controlled left thigh pain, after walking into a chair at home. This was determined to be a soft tissue injury.

She had previously attended the Outpatient Endocrinology Clinic for investigation of hypercalcaemia, which had a potential familial tendency, as her mother, daughter, and granddaughter also had documented hypercalcaemia. Her past medical history included a long history of hypercalcaemia, thyrotoxicosis currently treated with $20 \mathrm{mg}$ of carbimazole daily as well as previous thyroid surgery at seventeen years old, Type 2 Diabetes Mellitus treated with metformin, dyslipidaemia, and depression.
At admission, she had a number of symptoms attributable to hypercalcaemia including constipation, dry mouth, abdominal pains, and atraumatic fractures of the left wrist and left shoulder after a fall from standing height. She had no history of nephrolithiasis nor of cholelithiasis.

On examination, her general physical observations were normal and she was alert and orientated. Cardiovascular, respiratory, and abdominal examinations were normal. Examination of the left hip revealed no deformities. There was a small, $3 \mathrm{~cm}$ area of bruising over the left lateral thigh, which was tender on palpation. She was also tender over the left greater trochanter. The range of motion of the left hip was reduced and limited by pain on active and passive movement. On examination of the spine, she was tender over the C7 and T10 vertebral bodies. Lower limb examination showed reduced power of $3 / 5$ for left hip flexion and extension, and normal 5/5 power of the other myotomes.

The full blood count was normal, with a haemoglobin of $136 \mathrm{~g} / \mathrm{L}$, a white cell count of 5.7, and platelet count of 165 $\mathrm{x} 10^{\wedge} 9 / \mathrm{L}$. On admission, the serum calcium corrected for albumin was raised at $2.93 \mathrm{mmol} / \mathrm{L}$ (Ref range $2.15-2.55$ ) and the serum phosphate was normal at $0.92 \mathrm{mmol} / \mathrm{L}$ (Ref range $0.81-1.45$ ). During her hospital admission the serum calcium peaked at $3.04 \mathrm{mmol} / \mathrm{L}$. The ionised calcium was high at $1.79 \mathrm{mmol} / \mathrm{L}$ (Ref range 1.15-1.35). Her renal function was normal with a creatinine of $35 \mathrm{umol} / \mathrm{L}$ (Ref range 46 - 99) and estimated GFR of $>90 \mathrm{~mL} / \mathrm{min} / 1.73^{\wedge} 2$.

The parathyroid hormone was inappropriately normal at 3.0 $\mathrm{pmol} / \mathrm{L}$ (Ref range 1.0-7.0) and 25-Hydroxy-Vitamin D was normal at $97 \mathrm{mmol} / \mathrm{L}$ (Ref range 50-150). 
A Sestamibi Parathyroid Study showed no hyperfunctioning parathyroid adenoma. The Thyroid Pertechnetate Scan demonstrated increased activity in the remnant of the right thyroid lobe and isthmus, consistent with her history of thyrotoxicosis. No parathyroid adenoma was visualised on the Thyroid Ultrasound. Bone Mineral Densitometry of the lumbar spine and femoral neck was consistent with osteoporosis. CT of the chest, abdomen and pelvis was performed to investigate a malignant cause of her hypercalcaemia. This showed a small lytic area in the right iliac crest, which was unchanged in comparison to a previous CT from twelve months earlier. There was otherwise no other evidence of malignancy.

The calculated calcium to creatinine clearance ration was 0.0096 , indicating greater than $99 \%$ reabsorption of filtered calcium in spite of hypercalcaemia (Fig. 2).

To confirm a diagnosis of FHH, molecular genetic analysis for CASR was performed using bi-directional Sanger sequencing of PCR amplified genomic DNA encompassing CASR exons 2-7, including splice donor and acceptor sites. This detected a heterozygous mutation for c.554G>A p. (Arg185Gln), a known inactivating missense mutation of CASR. This was consistent with a diagnosis of FHH.

Figure 1: Pedigree of the case. Squares represent male family members. Circles represent female family members. Clinical status is indicated by a solid symbol (affected), and an open symbol (unaffected).

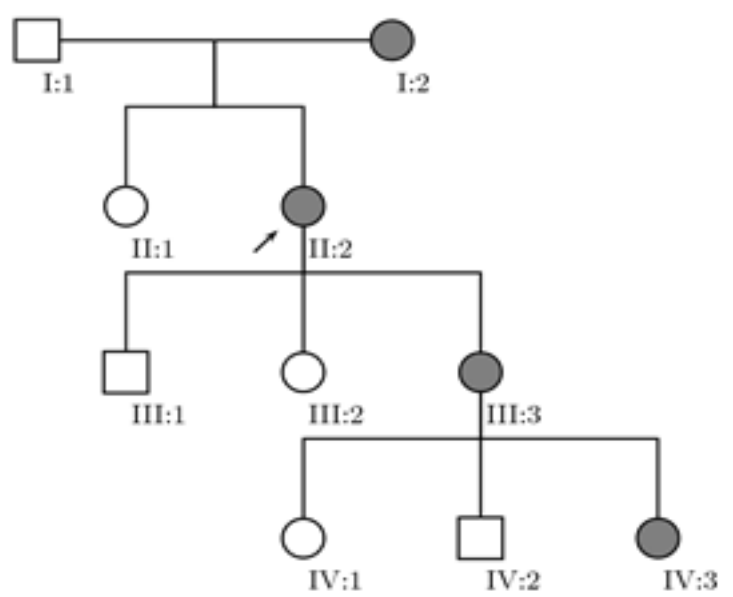

Figure 2: Calculation for the calcium to creatinine clearance ration. To exclude Familial Hypocalciuric Hypercalcaemia (FHH), Ca: Cr ratio should be $>0.01$

$\mathrm{Ca} / \mathrm{Cr}$ clearance ration $=$ Urine $\mathrm{Ca}(\mathrm{mmol} / \mathrm{L}) \times[$ Serum $\mathrm{Ca}$ (umol/L)/1000]

Seurm Ca $(\mathrm{mmol} / \mathrm{L})$ x Urine $\mathrm{Cr}(\mathrm{mmol} / \mathrm{L})$

$=0.8 \times(33 / 1000)$

$3.01 \times 0.7$

$=0.0096$
While awaiting further diagnostic studies, empiric management for hypercalcaemia was commenced. This patient received generous IV fluid replacement and ultimately bisphosphonate therapy, administered as IV pamidronate $60 \mathrm{mg}$ over 4 hours which appropriately led to normalisation of serum calcium. During this period her analgesia was titrated to effect and ultimately her mobility was adequate for safe discharge home. She is being followed up as an outpatient.

\section{Discussion}

Hypercalcemia is a relatively common clinical problem and the most common causes are PHPT and malignancy. FHH is a hypercalcemic syndrome usually characterized by as an asymptomatic and uncomplicated hypercalcemia and apparent normal longevity.

When PTH is only minimally elevated or within the normal range (but inappropriately normal given the patient's hypercalcemia), PHPT remains the most likely diagnosis, although FHH is possible. ${ }^{[5]}$

In FBHH, 1, 25-dihydroxyvitamin $\mathrm{D}$ levels have been reported to be normal. ${ }^{[6]}$

Renal cell carcinoma often shows hypercalcemia, so we evaluated the relationship between renal cell carcinoma and hypercalcemia in this case. The radiologic findings revealed no evidence of distant metastasis including bone or local recurrence.

Kidney function, is normal, as well as urinary concentrating ability, in contrast to hyperparathyroidism. ${ }^{[7]}$

There are more than 40 inactivating mutations in the CaSR gene have been identified in patients with $\mathrm{FHH} .^{[8]}$

\section{Conclusions}

FHH is a benign condition and included in differential diagnosis of hypercalcaemia with normal or elevated PTH. CCCR is used to distinguish it from PHPT, however the definitive diagnosis demands genetic study, in order to avoid needless parathyroidectomy. In this patient, a novel mutation in CASR gene was identified.

\section{Author Contributions}

Tivya Kulasegaran - Substantial contributions to conception and design, Acquisition of data, Analysis and interpretation of data, Drafting the article, revising it critically for important intellectual content, Final approval of the version to be published

Pranav Kumar - Substantial contributions to conception and design, Acquisition of data, Analysis and interpretation of data, Drafting the article, Revising it critically for 
important intellectual content, Final approval of the version to be published

\section{Guarantor of Submission}

The corresponding author is the guarantor of submission.

\section{Source of Support}

None

\section{Consent Statement}

Written informed consent was obtained from the patient for publication of this case report.

\section{Conflict of Interest}

Authors declare no conflict of interest.

\section{References}

[1] Zhang C, Miller CL, Brown EM, Yang JJ. The calcium sensing receptor: from calcium sensing to signaling. Sci China Life Sci. 2015; 58:14-27.

[2] Mrgan M, Nielsen S, Brixen K. Familial hypocalciuric hypercalcemia and calcium sensing receptor. Acta Clin Croat. 2014; 53:220-225.

[3] Christensen SE, Nissen PH, Vestergaard P, Mosekilde L. Familial hypocalciuric hypercalcaemia: a review. Curr Opin Endocrinol Diabetes Obes. 2011; 18:359-370.

[4] Law WM Jr, Heath H 3rd. Familial benign hypercalcemia (hypocalciuric hypercalcemia). Clinical and pathogenetic studies in 21 families. Ann Intern Med. 1985; 102:511-519.

[5] Eldeiry LS, Ruan DT, Brown EM, Gaglia JL, Garber JR. Primary hyperparathyroidism and familial hypocalciuric hypercalcemia: relationships and clinical implications. Endocr Pract. 2012;18:412-417

[6] Law WM, Jr, Heath H., 3rd Familial benign hypercalcemia (hypocalciuric hypercalcemia). Clinical and pathogenetic studies in 21 families. Ann Intern Med. 1985; 102:511-519.

[7] Marx SJ, Attie MF, Stock JL, Speigel AM, Levine MA. Maximal urine-concentrating ability: familial hypocalciuric hypercalcemia versus typical primary hyperparathyroidism. J Clin Endocrinol Metab.1981; 52:736-740.

[8] $\mathrm{Hu}$ J, Spiegel AM. Naturally occurring mutations of the extracellular $\mathrm{Ca} 2+-$-sensing receptor: implications for its structure and function. Trends Endocrinol Metab. 2003; 14:282-288. 